Supporting Information: QIIME v1.9 Protocol

\title{
The Microbiome of Size-Fractionated Airborne Particles from the Sahara Region
}

Rebecca A. Stern ${ }^{\mathrm{a}^{*}}$, Nagissa Mahmoudi ${ }^{\mathrm{b}}$, Caroline O. Buckee ${ }^{\mathrm{c}}$, Amina T. Schartup $^{\mathrm{d}, \mathrm{e}}$, Petros Koutrakis ${ }^{\mathrm{d}}$, Stephen T. Ferguson ${ }^{d}$, Jack Mikhail Wolfson ${ }^{d}$, Steven C. Wofsy ${ }^{\mathrm{f}}$, Bruce C. Daube ${ }^{\mathrm{f}}$, Elsie M. Sunderland ${ }^{\mathrm{a}, \mathrm{d}}$

${ }^{a}$ Harvard John A. Paulson School of Engineering and Applied Science, Harvard University, Cambridge, MA, USA 02138

bDepartment of Earth and Planetary Sciences, McGill University, Montreal, QC, H3A 0E8

${ }^{c}$ Center for Communicable Disease Dynamics, Department of Epidemiology, Harvard T.H. Chan School of Public Health, Boston, MA 02115

dDepartment of Environmental Health, Harvard T.H. Chan School of Public Health, Boston, MA, 02115

eScripps Institution of Oceanography, La Jolla, CA 92037

fDepartment of Earth and Planetary Sciences, Harvard University, Cambridge, MA 02138

*Rebecca_stern@g.harvard.edu 
Sequences were processed using QIIME v1.91 following Mahmoudi et al. (2015)² with modifications.

Individual steps are as follows:

Extracted barcodes that indicated the sample ID ( 8 base pairs):

extract_barcodes.py -f inseqs.fastq -c barcode_single_end --bc1_len 8 -bc2_len 0 -o processed_seqs

Assembled and aligned the reads using the QIIME script join_paired_ends.py using the fastq_join method:

join_paired_ends.py -f \$PWD/forward_reads.fastq -m fastq_join -r

\$PWD/reverse_reads.fastq -o \$PWD/fastq-join_joined

Extracted barcodes to remove the primers:

extract_barcodes.py -f inseqs.fastq--bc1_len 15 --bc2_len 18 -c

barcode_paired_stitched -o processed_seqs

Loaded in the mapping file and verified it:

validate_mapping_file.py -m Fasting_Map.txt -o

validate_mapping_file_output

Demultiplexed and quality filtered the sequence data (separated the reads into sample IDs and removed low quality sequences). The quality filter was set to a Phred score of 20 as the maximum unacceptable Phred quality score.

split_libraries_fastq.py -i lanel_readl.fastq.gz -b lanel_barcode.fastq.gz --rev_comp_mappīng_barcodes -o slout_q20/ -m map.txt -q $1 \overline{9}$

Checked for chimeras using the .fna file generated from the previous step using USEARCH61. ${ }^{3}$

identify_chimeric_seqs.py -i repr_set_seqs.fasta -t

taxonomy_assignmeñt.txt $-r$ ref_seq_set.fna -m usearch61-split_by_sampleid -o chimeric_seqs_blast.txt

Filtered out chimeric sequences.

filter_fasta.py -f seqs.fna -o seqs_chimera_filtered -s chimeras.txt -n

Taking the clean, assembled reads with barcodes/primers removed from the preceding steps, we next did OTU picking. We picked OTUs at $99 \%$ similarity and assigned taxonomy using the RDP classifier ${ }^{4}$ against the Greengenes (v 13_8) database..$^{5}$ The parameters file contents are provided at the bottom of this workflow in blue font.

pick_open_reference_otus.py -i \$PWD/seqs1.fna -r \$PWD/refseqs.fna -o

\$PWD/ucrss_sortmernā_sumaclust/ -p \$PWD/qiime_parameters.txt -m

sortmerna_sumaclust

We removed OTUs that comprised less than $0.005 \%$ of the total data set to limit the effect of spurious OTUs on analysis. ${ }^{6,7}$ 
filter otus from otu table.py -i

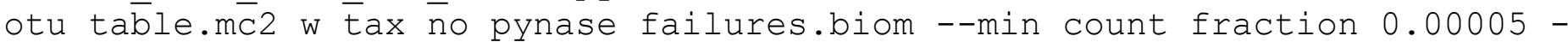

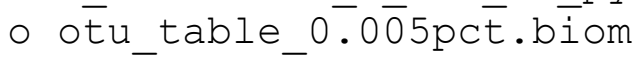

We calculated the minimum number of sequences needed to rarefy by using:

biom summarize-table -i otu table 0.005pct.biom -o

cmed_stats_otu_table_0.005p čt_remōved

We ran a set of core diversity analyses and rarefied by the minimum number of sequences (27680) in the output of the preceding step.

core_diversity_analyses.py -i \$PWD/otu_table0.005pct.biom -o

\$PWD/core_output -m \$PWD/map.txt -c SampleType, day -t \$PWD/rep_set.tre -e 20

Within the core diversity generated products, we used the collated alpha diversity files for processing in Python (Dunn's test).

We ran Kruskal Wallis tests using the QIIME script:

group significance.py -i otu table.biom -m map overlapping.txt -c diet -s kruskäl_wallis -o gtest_ocs. txxt

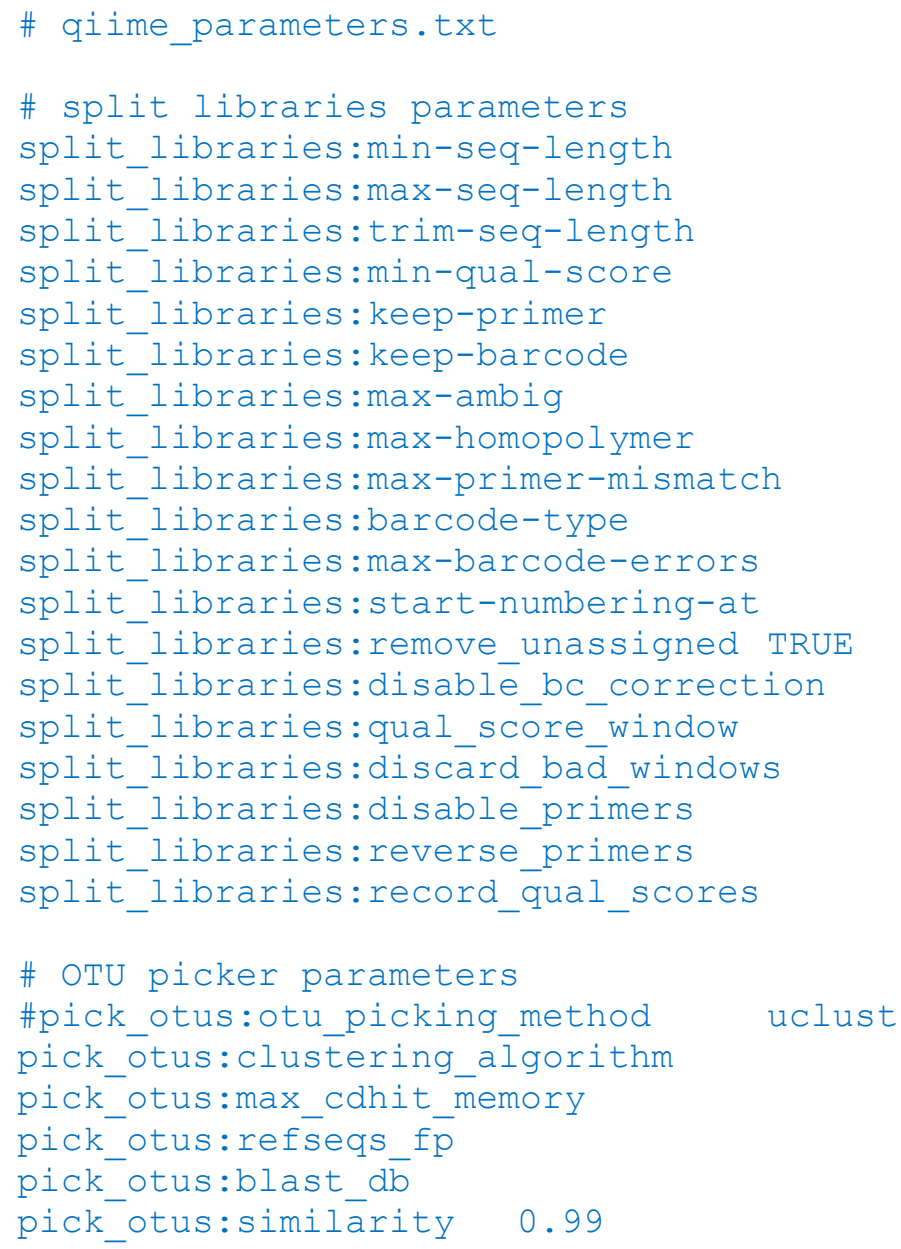




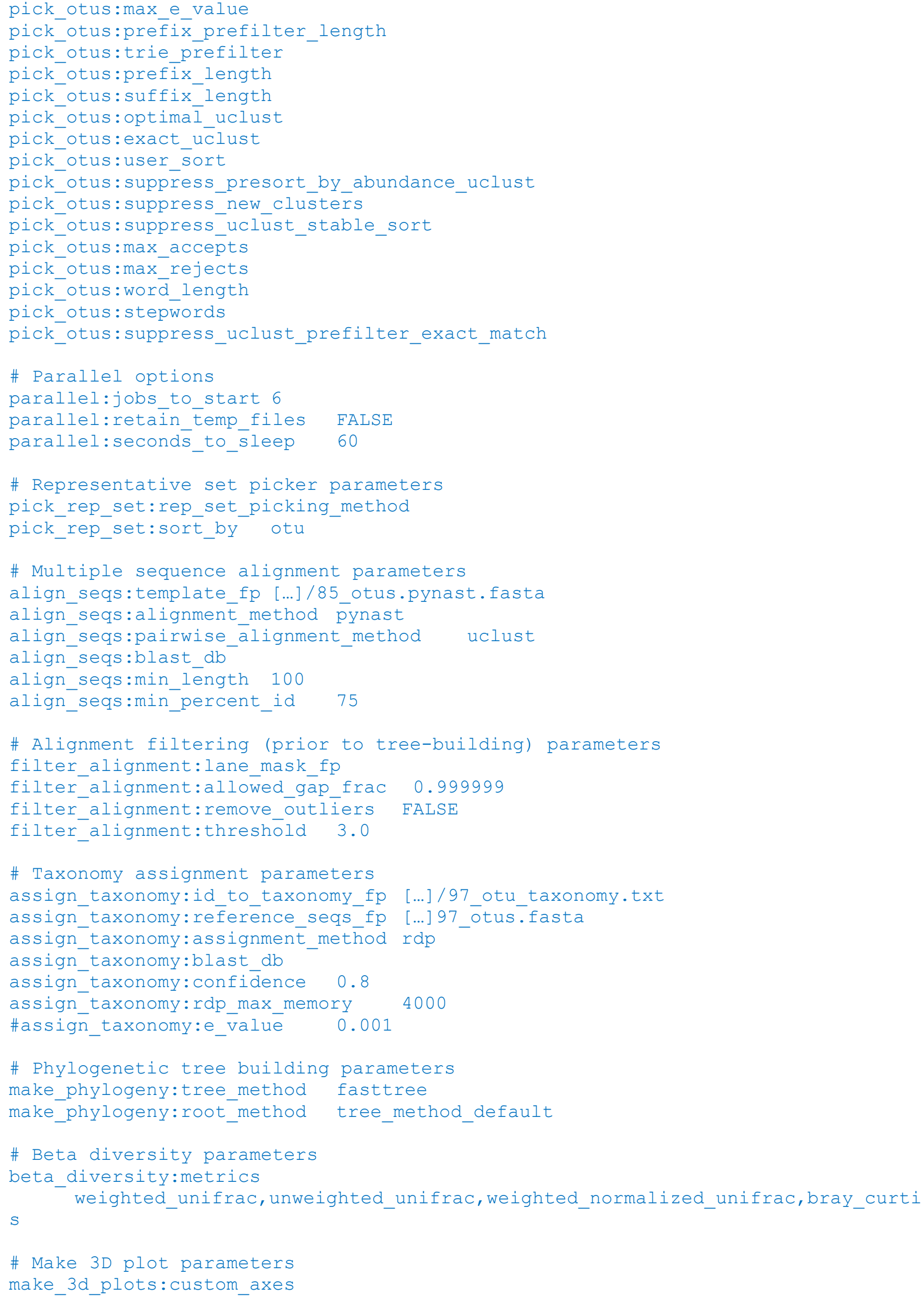




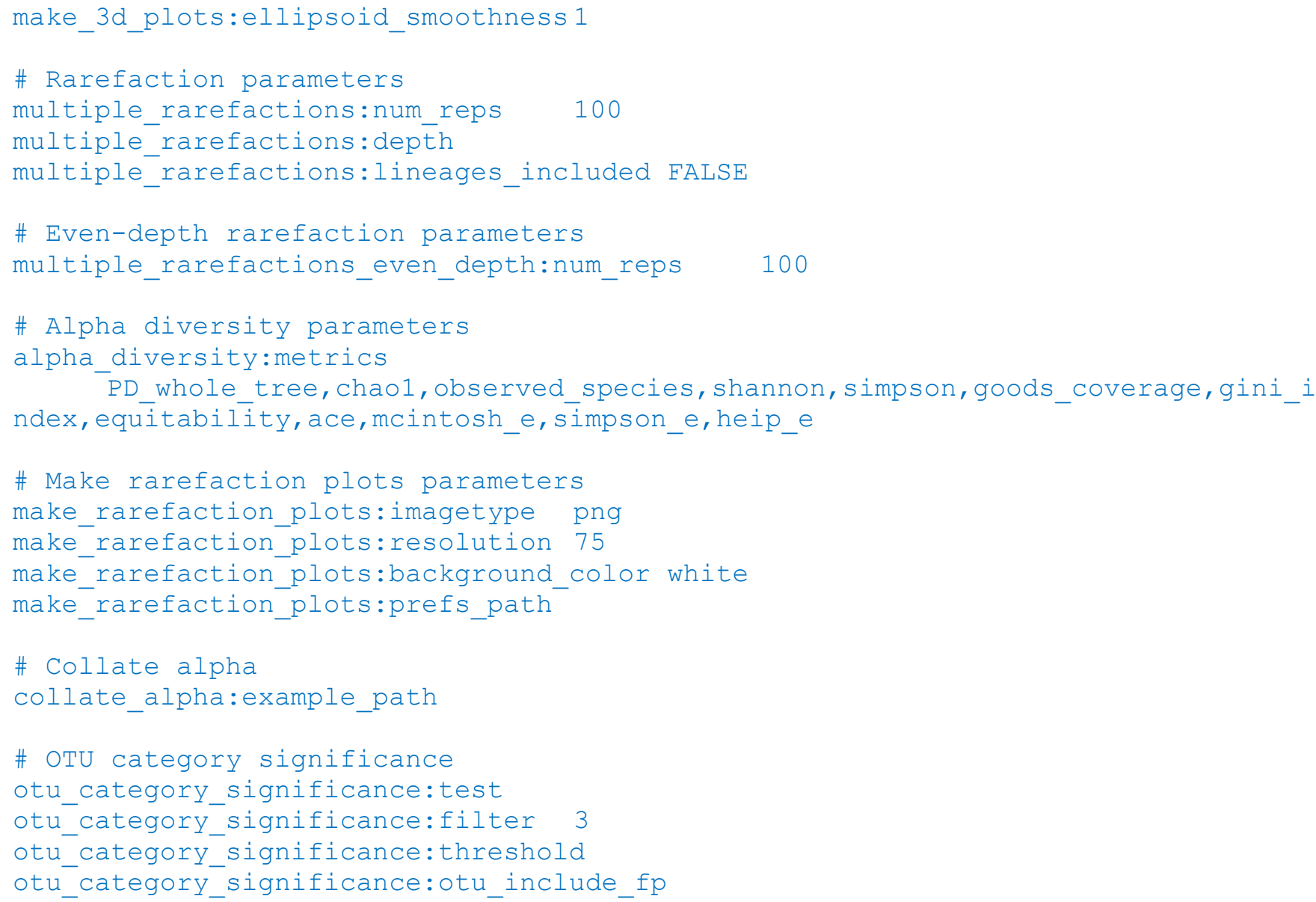

\section{References:}

(1) Caporaso, J. G.; Kuczynski, J.; Stombaugh, J.; Bittinger, K.; Bushman, F. D.; Costello, E. K.; Fierer, N.; Pẽa, A. G.; Goodrich, J. K.; Gordon, J. I.; Huttley, G. A.; Kelley, S. T.; Knights, D.; Koenig, J. E.; Ley, R. E.; Lozupone, C. A.; McDonald, D.; Muegge, B. D.; Pirrung, M.; Reeder, J.; Sevinsky, J. R.; Turnbaugh, P. J.; Walters, W. A.; Widmann, J.; Yatsunenko, T.; Zaneveld, J.; Knight, R. QIIME Allows Analysis of High-Throughput Community Sequencing Data. Nat. Methods 2010, 7 (5), 335-336. https://doi.org/10.1038/nmeth.f.303.

(2) Mahmoudi, N.; Robeson, M. S.; II, H. F. C.; Fortney, J. L.; Techtmann, S. M.; Joyner, D. C.; Hazen, T. C. Microbial Community Composition and Diversity in Caspian Sea Sediments. FEMS Microbiol. Ecol. 2015, 91 (1), 1.

(3) Edgar, R. C. Search and Clustering Orders of Magnitude Faster than BLAST. Bioinformatics 2010, 26 (19), 2460-2461. https://doi.org/10.1093/bioinformatics/btq461.

(4) Wang, Q.; Garrity, G. M.; Tiedje, J. M.; Cole, J. R. Naïve Bayesian Classifier for Rapid Assignment of RRNA Sequences into the New Bacterial Taxonomy. Appl. Environ. Microbiol. 2007, 73 (16), 52615267. https://doi.org/10.1128/AEM.00062-07.

(5) McDonald, D.; Price, M. N.; Goodrich, J.; Nawrocki, E. P.; Desantis, T. Z.; Probst, A.; Andersen, G. L.; Knight, R.; Hugenholtz, P. An Improved Greengenes Taxonomy with Explicit Ranks for Ecological and Evolutionary Analyses of Bacteria and Archaea. ISME J. 2012, 6 (3), 610-618. https://doi.org/10.1038/ismej.2011.139.

(6) Bokulich, N. A.; Subramanian, S.; Faith, J. J.; Gevers, D.; Gordon, J. I.; Knight, R.; Caporaso, J. G. Quality-Filtering Vastly Improves Diversity Estimates from Illumina Amplicon Sequencing. Nat. Methods 2013, 10 (1), 57-59.

(7) Navas-Molina, J. A.; Peralta-Sánchez, J. M.; González, A.; McMurdie, P. J.; Vázquez-Baeza, Y.; Xu, Z.; Ursell, L. K.; Lauber, C.; Zhou, H.; Song, S. J.; Huntley, J.; Ackermann, G. L.; Berg-Lyons, D.; Holmes, S.; Caporaso, J. G.; Knight, R. Advancing Our Understanding of the Human Microbiome Using QIIME, 
1st ed.; Elsevier Inc., 2013; Vol. 531. https://doi.org/10.1016/B978-0-12-407863-5.00019-8. 